\title{
A Miniaturized Two Axis Sun Sensor for Attitude Control of Nano-Satellites
}

\author{
Pablo Ortega, Gema López-Rodríguez, Jordi Ricart, Manuel Domínguez, Luis M. Castañer, Senior Member, IEEE, \\ José M. Quero, Senior Member, IEEE, Cristina L. Tarrida, Juan García, Manuel Reina, Ana Gras, and \\ Manuel Angulo
}

\begin{abstract}
This paper describes the design, fabrication, characterization, and satellite integration of a miniaturized two axis sun sensor which has been used in the attitude control system of the Spanish nano-satellite NANOSAT-1B. This device is made of four silicon photodiodes monolithically integrated in a crystalline silicon substrate, protected by a transparent cover glass assembled on the same silicon die against space radiation damage. The sensor fabrication combines standard silicon processing technology with a high performance solar cell fabrication process. The sensor, including electronics and mechanical and electrical interfacing with the satellite, has a small size $(3 \mathrm{~cm} \times 3 \mathrm{~cm})$ and low weight $(24 \mathrm{gr}$.), with a sun field-of-view greater than $\pm 60^{\circ}$ with an angle accuracy better than $0.15^{\circ}$. Three of these sensors have already been integrated in the NANOSAT-1B platform that has been successfully launched in July 2009.
\end{abstract}

Index Terms-Angle measurement, cover glass, field-of-view (FOV), satellite attitude control, silicon photodiodes, sun sensor.

\section{INTRODUCTION}

$\mathbf{T}$ HERE is an increasing number of satellites orbiting the Earth for many applications. Although originally used for military purposes, today satellites are now also responsible for weather surveillance, scientific measurements, telecommunications, and highly accurate global positioning systems (GPSs). All these platforms need attitude control systems to ensure that instruments, solar panels, antennas and other hardware are properly oriented to perform their duties. Controlling attitude requires sensors, to measure satellite attitude, and actuators to apply the torques needed to reorient the platform to a desired position. Thrusters, reaction or momentum wheels, magnetotorque coils, and gravity gradient torques are used typically as

Manuscript received January 20, 2010; revised March 22, 2010; accepted March 23, 2010. Date of publication June 10, 2010; date of current version August 04, 2010. This work was supported in part by the Spanish Government under Program ESP2007-299975-E. The associate editor coordinating the review of this paper and approving it for publication was Prof. Evgeny Katz.

P. Ortega, G. López-Rodríguez, J. Ricart, M. Domínguez, and L. M. Castañer are with the Universidad Politécnica de Cataluña (UPC), Grupo de Micro y Nanotecnologías, Departamento de Ingeniería Electrónica, E-08034 Barcelona, Spain (e-mail: ortega@eel.upc.edu; glopez@eel.upc.edu; ricart@eel.upc.edu; mpumar@eel.upc.edu; castaner@eel.upc.edu).

J. M. Quero, C. L. Tarrida, and J. García are with the Departamento de Ingeniería Electrónica, Escuela Superior de Ingenieros, Universidad de Sevilla (US), 41092 Sevilla, Spain (e-mail: quero@us.es; tarrida@zipi.us.es; juangar@zipi.us.es).

M. Reina, A. Gras, and M. Angulo are with the Instituto Nacional de Técnica Aeroespacial (INTA), 28850 Torrejón de Ardoz, Madrid, Spain (e-mail: reinam@inta.es; grassa@inta.es; angulom@inta.es).

Color versions of one or more of the figures in this paper are available online at http://ieeexplore.ieee.org.

Digital Object Identifier 10.1109/JSEN.2010.2047104 actuators [1]-[4]. Gyroscopes, horizon earth detectors, magnetometers, earth gravity sensors, infrared earth radiation detection, or star trackers are commonly used as attitude sensors [5]-[7]. Even more popular are sun sensors that can determine the attitude by measuring the sun vector [8]-[10].

Sun sensors are nowadays successfully used in terrestrial applications, especially in the field of renewable energies as, for example, concentration photovoltaic plants and heliostats, where sun tracking is mandatory to maximize conversion efficiency [11]. In these cases the devices require a small value of the field-of-view (FOV), typically $\pm 10^{\circ}$, with very accurate angle measurement (precision about $0.01^{\circ}$ ). In satellite applications, a common requirement is to measure the sun vector in any position inside a $360^{\circ}$ range around the platform. This requirement is usually fulfilled using three sensors with $120^{\circ} \mathrm{FOV}\left( \pm 60^{\circ}\right)$ working together. However, a wider FOV in classical sun sensors involves a worse precision. Sun sensors in satellite applications have typically resolutions about $0.5^{\circ}$ and algorithms to discriminate solar reflection in the earth (albedo component). Commercial light angle sensors [12] or related devices [13] are susceptible to be used as sun sensors, but their application in space is prevented by the harsh conditions of the environment due to radiation and extreme temperatures. Besides, small size, low weight and low power consumption are also mandatory. Although some attempts of sun sensors application to space can be found in the literature, the fact is that either, they have relatively large sizes $\left(>10 \mathrm{~cm}^{2}\right)$, hence incompatible with small platforms (nano, and pico-satellites) [8], or fail to meet the mission requirements such as the micro-optical-mechanical systems (MOEMS) [9], [10] based devices, which do not provide experimental accuracies better than $0.5^{\circ}$ in the whole FOV range.

In this paper, we describe a miniaturized two axis sun sensor with a $\pm 60^{\circ} \mathrm{FOV}$ and an angle accuracy better than $0.5^{\circ}$. Both parameters are defined by satellite manufacturer (INTA) in order to fulfill the NANOSAT-1B mission requirements. The sensor combines standard silicon processing technology with a UPC baseline crystalline silicon solar cell fabrication process, leading to small area $\left(<10 \mathrm{~cm}^{2}\right)$, low weight $(<20 \mathrm{gr})$, low cost, and low-power device. All materials used in the sensor fabrication process are compatible with space requirements in terms of thermal and vibration resistance, and low degasification (ECSS-70-71A rev.1 and ECSS-Q-70-08A standards [14], [15]). Furthermore the device incorporates on top of the chip a transparent radiation shield, called cover-glass, traditionally used as radiation protection in space photovoltaic modules and cells [16]. Three of these sensors have already been integrated 


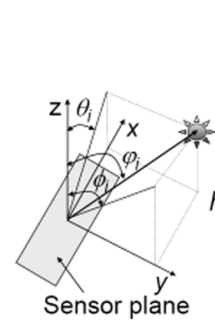

(a)

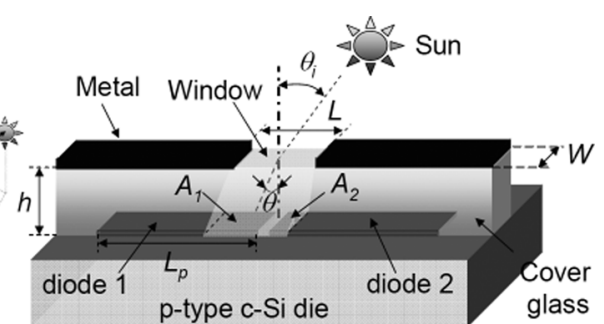

(b)
Fig. 1. (a) Definition of the two sun vector angles $\theta_{i}$ and $\phi_{i}$. (b) A simplified scheme of a pair of photodiodes to measure one particular sun incidence angle (in this case angle $\theta_{i}$ ).

in the Spanish nano-satellite NANOSAT-1B that has been launched in July of 2009. This satellite has a small size (less than half a meter side with a weight less than $20 \mathrm{Kg}$ ) and it has been placed in a sun-synchronus orbit, $660 \mathrm{Km}$ altitude (low earth orbit), $98^{\circ}$ inclination angle, 98 min orbital rotation period, and a mission life between three and five years. The main purpose of NANOSAT platform series is the demonstration in orbit of new emerging technologies with scientific payloads, for observation or communications using low weight and low-cost integrated instruments.

\section{SENSOR ConcePt AND DeVICE Design}

\section{A. Concept}

The sensor consists of two pairs of photodiodes fabricated monolithically in the same crystalline silicon substrate and placed orthogonally to measure the two angles of the sun vector defined as it can be seen in Fig. 1(a). The FOV is restricted in a cone of $\varphi_{i}= \pm \varphi_{i \max }$, and therefore, using basics trigonometry, angles $\theta_{i}$ and $\phi_{i}$ must satisfy (1)

$$
t g^{2}\left(\theta_{i}\right)+t g^{2}\left(\phi_{i}\right)=t g^{2}\left(\varphi_{i}\right) \leq t g^{2}\left(\varphi_{i \max }\right) .
$$

To measure one particular angle we use a pair of photodiodes using the simplified scheme depicted in Fig. 1(b). The sensor concept is similar to a previous device reported by us in [11], but in this case the device integrates on chip a transparent glass (cover glass) of thickness $h$ that is metalized in the top surface with the exception of a small window of length $L$ and width $W$. The sun light illuminates the diodes partially through this window, being $A_{1}$ and $A_{2}$ the illuminated areas of photodiodes 1 and 2 , respectively. In our sensor, instead of using a conventional glass cover, we have performed the metallization directly on a space cover glass acting additionally as a shield for extraterrestrial radiation. The impinging sun radiation generates a photocurrent in each photodiode, which is proportional to the projected irradiance in the sensor plane and to the illuminated area [11]. This area depends of the light angle in the glass $\theta$, that differs from incident light angle $\theta_{i}$ due to refraction in the glass-air interface. Refracted angle $\varphi$ is related with incident angle $\varphi_{i}$ by the well known Snell's law (2), where $n_{c g}$ and $n_{\text {air }}$ are the refraction index of cover glass $\left(n_{c g}=1.49\right)$ and air $\left(n_{\text {air }}=1\right)$, respectively

$$
n_{\text {air }} \cdot \operatorname{sen}\left(\varphi_{i}\right)=n_{c g} \cdot \operatorname{sen}(\varphi)
$$

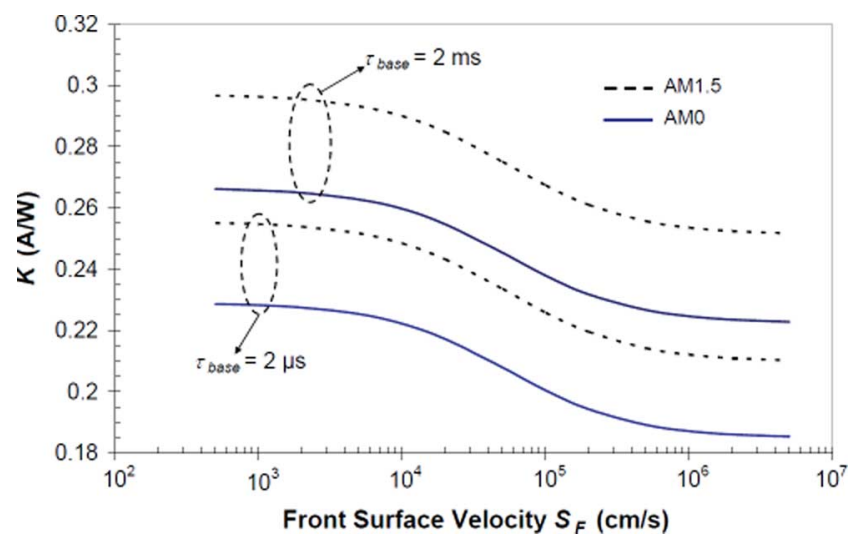

Fig. 2. $K$ estimation using PC-1D simulator semiconductor program under AM0 and AM1.5 standard solar spectrums. Curves are obtained for different front surface velocities $S_{F}$ and parameterized for two representative base lifetimes.

When the sun is located just in the direction normal to the photodiode plane $\left(\varphi_{i}=0^{\circ}\right)$, the illuminated areas $A_{1}$ and $A_{2}$ are the same, and so the photocurrents are equal provided the photodiode characteristics have a good match. For an arbitrary relative position between the sun vector and the photodiode plane, the illuminated areas will be different and therefore photocurrents too. Photocurrents in a photodiode pair (for instance to determine $\theta$ angle) can be calculated as follows:

$$
\begin{aligned}
I_{f_{1}} & =K \cdot A_{1}(\theta) \cdot S_{i n}\left(\varphi_{i}\right) \\
& =K \cdot W \cdot\left(\frac{L}{2}+t g(\theta) \cdot h\right) \cdot \cos \left(\varphi_{i}\right) \cdot S C \cdot \varepsilon \\
I_{f_{2}} & =K \cdot A_{2}(\theta) \cdot S_{i n}\left(\varphi_{i}\right) \\
& =K \cdot W \cdot\left(\frac{L}{2}-t g(\theta) \cdot h\right) \cdot \cos \left(\varphi_{i}\right) \cdot S C \cdot \varepsilon .
\end{aligned}
$$

The irradiance normal to the incidence plane $S_{i n}$ is related to the solar constant $\mathrm{SC}\left(1366 \mathrm{~W} / \mathrm{m}^{2}\right)$, the incidence angle $\varphi_{i}$, and the earth orbit eccentricity $\varepsilon$, that varies along the year. The parameter $K$ determines the incident power to current conversion in units of A/W. Ideally, $K$ takes the same value in the two photodiodes, and it depends strongly on the fabrication process (antireflection coating, front and rear surface passivation, bulk lifetime, etc.). So its value changes with temperature, sun light spectrum (AM0 in space applications), incidence angle, and extraterrestrial radiation dose. An estimation of the $K$ parameter for AM0 and AM1.5 spectrums is shown in Fig. 2. These curves were obtained using the semiconductor simulation program PC1D [17] taking into account the front surface recombination by means of the front surface recombination velocity $S_{F}$. Curves are parameterized for two extreme base lifetimes (volume recombination), $\tau_{\text {base }}, 2 \mu$ s and $2 \mathrm{~ms}$.

Higher $\tau_{\text {base }}$ and lower $S_{F}$ values can be representative at the beginning of the mission beginning of life (BOL), and the lower lifetime and higher $S_{F}$ values for the final time end of life (EOL) as consequence of material physical degradation by space radiation in one device with a nonefficient radiation shield. A nominal BOL $K$ value of $0.26 \mathrm{~A} / \mathrm{W}$ is expected in our devices under AM0 spectrum (or 0.29 A/W under terrestrial AM1.5 spectrum), assuming a $S_{F} \sim 10^{4} \mathrm{~cm} / \mathrm{s}$ and $\tau_{\text {base }}=2 \mathrm{~ms}$. As can be seen 


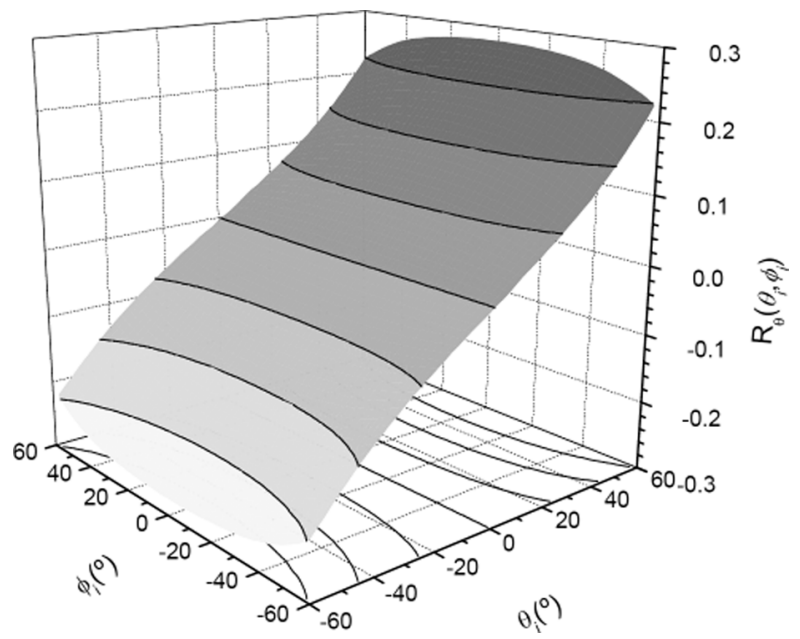

Fig. 3. Theoretical dependence of $R_{\theta}$ with the incident angles $\theta_{i}$ and $\phi_{i}$ considering a geometrical gain of $G=0.4(L=2 \mathrm{~mm}$ and $h=400 \mu \mathrm{m})$.

in Fig. 2 the values of $K$ can strongly depend not only on the physical and technological material parameters, but also on the time of the day (angle dependence) and on the mission life (radiation dose). This is why the sensor cannot rely on the absolute values of the photocurrents but instead on a 'relative differential' function $R_{\theta}$, defined as

$$
R_{\theta}=\frac{I_{f 1}-I_{f 2}}{I_{f 1}+I_{f 2}}=\frac{2 h \cdot t g(\theta)}{L}=G \cdot \operatorname{tg}(\theta) \text { with } 0 \leq\left|R_{\theta}\right| \leq 1
$$

where $G$ is defined as a geometrical gain $(2 h / L)$. From (4), as expected, it can be seen that the function $R_{\theta}$ is proportional to $G$, and therefore, a thicker cover glass improves the angle sensitivity but jeopardizes sensor size. The same strategy can be used to define a similar function for angle $\phi$, as follows:

$$
R_{\phi}=\frac{I_{f 3}-I_{f 4}}{I_{f 3}+I_{f 4}}=\frac{2 h \cdot t g(\phi)}{L}=G \cdot \operatorname{tg}(\phi) .
$$

It is important to remind that the refracted angles $\theta$ and $\phi$ depend on both incident angles $\theta_{i}$ and $\phi_{i}$. Therefore, $R_{\theta}$ and $R_{\phi}$ are, in fact, two variable functions of $\theta_{i}$ and $\phi_{i}$. As an example, theoretical values of the function $R_{\theta}\left(\theta_{i}, \phi_{i}\right)$ are plotted in Fig. 3 for given geometrical values. The full determination of $\theta_{i}$ and $\phi_{i}$ needs a correlation of both functions $R_{\theta}$ and $R_{\phi}$

\section{B. Design}

The sun sensor has been designed to satisfy the NANOSAT-1B mission requirements, that means, an angle accuracy beteer than $0.5^{\circ}$ and a minimum FOV of $\varphi_{i \max }= \pm 60^{\circ}$, or using (2), a transmitted range angle in the glass of $\varphi_{\max }=$ $\pm 36^{\circ}$. Therefore, the photodiode and window lengths, $L_{p}$ and $L$ respectively (see Fig. 1), should be long enough to accommodate the light spot in the active solar cell region in a worst case of incidence angle $\left(\phi=0^{\circ}\right.$ and $\left.\theta=\theta_{\lim }= \pm 36^{\circ}\right)$ according to

$$
L_{p} \geq L>2 h \cdot \operatorname{tg}\left(\theta_{\lim }\right) .
$$

Taking into account the available commercial cover glass thicknesses (below $0.5 \mathrm{~mm}$ ), a minimum window length about

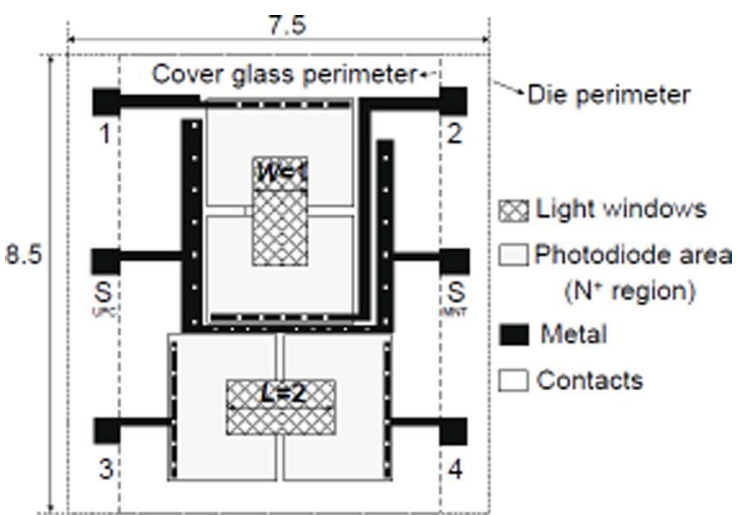

(a)

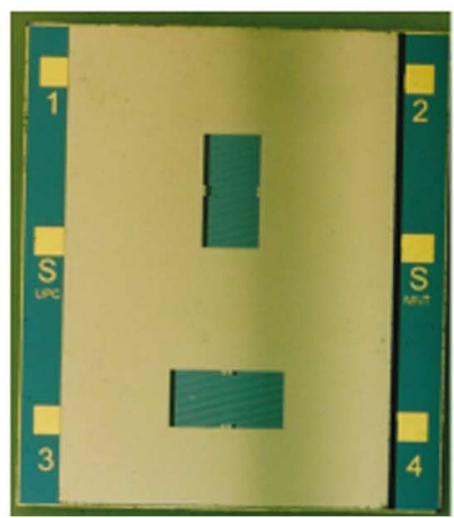

(b)

Fig. 4. (a) Two axis sun sensor layout. Dimensions are in $\mathrm{mm}$. Contour in dashed line indicates where the cover glass will be placed onto the silicon die. (b) An image of a fabricated silicon die with the two pair of photodiodes and the cover glass integrated on the same substrate. Emitter or cathodes electrodes (pads labeled 1-4) and base or anode electrodes (two redundant electrodes labeled S "Substrate") are easily distinguished in the image. At the center of the each window we can see four small features to perform the fine alignment between cover glass and the silicon substrate.

$0.75 \mathrm{~mm}$ is necessary. However, $L$ was oversized to $2 \mathrm{~mm}$ to ensure that the window misalignment with the photodiodes does not bring significant inaccuracy to the measure. We assume $50 \mu \mathrm{m}$ as a maximum window misalignment (or a $\pm 5 \%$ of uncertainty in the solar cell area under perpendicular incidence). Moreover, the $2 \mathrm{~mm}$ window length value provides large enough current levels in the whole angle measurement range, leading to a high signal-to-noise ratio, and minimizing the impact of nonideal effects of the auxiliary electronics as can be voltage and/or currents offsets in the transimpedance preamplifier stage (see Section IV). For the same reasons, the window width was established to $W=L / 2(1 \mathrm{~mm})$, so the nominal solar cell area (under perpendicular sun incidence) is $1 \mathrm{~mm}^{2}$.

The layout used in this work along with a fabricated silicon device are shown in Fig. 4. The total silicon die area is $0.75 \mathrm{~cm} \times 0.85 \mathrm{~cm}$. The cover glass has a smaller width than the silicon substrate to allow the wire-bonding in the periphery. The electrode pads were also used to prealign the cover glass to the silicon die. The window placement and the distance between the photodiode pairs were calculated to avoid light coupling between them in the whole angle range of measurement. 


\section{DEVICE FABRICATION AND CHARACTERIZATION}

The fabrication of the silicon device has been done in the Laboratory of the MNT group of the UPC. The sensor fabrication combines standard processes used in silicon technology, with processing currently used at the MNT group to build high performance photovoltaic devices and solar cells [18]. The device fabrication sequence has three main stages: silicon die fabrication, cover glass metallization defining the two light windows, and cover glass bonding to the silicon die.

\section{A. Silicon Die Fabrication}

The silicon die is fabricated using $\mathrm{Fz}$ p-type $(1.0 \Omega \cdot \mathrm{cm})$ $\langle 100\rangle$ crystalline silicon 4 inch double side polished wafers. The fabrication process has the following steps: a) Thermal oxidation at $1060^{\circ} \mathrm{C} \mathrm{t}=240 \mathrm{~min}$. b) Definition of the emitter regions $\left(\mathrm{N}^{+}\right.$regions) of the four photodiodes using standard photolithography, $\mathrm{SiO}_{2}$ etching, and phosphorus doping using planar diffusion sources, $\mathrm{T}=870^{\circ} \mathrm{C} t=30 \mathrm{~min}$. c) $\mathrm{SiO}_{2}$ etching at the front and rear surfaces. d) Thermal dry oxidation for surface passivation (low recombination at surfaces) and antireflection coating purposes $\mathrm{T}=1070^{\circ} \mathrm{C} t=200 \mathrm{~min}$. e) Photolithography to define base contacts ( $\mathrm{P}+$ regions), $\mathrm{SiO}_{2}$ etching and boron diffusion using planar diffusion sources $\mathrm{T}=825^{\circ} \mathrm{C} t=40 \mathrm{~min}$. f) Emitter contacts in $\mathrm{N}+$ regions defined by photolithography and $\mathrm{SiO}_{2}$ etching. g) Finally, $\mathrm{Ti} / \mathrm{Pt} / \mathrm{Au}(30 \mathrm{~nm} / 120 \mathrm{~nm} / 350 \mathrm{~nm})$ deposition using sputtering and patterning using liftoff technique. A thermal annealing was performed at $\mathrm{T}=370^{\circ} \mathrm{C}$ during $20 \mathrm{~min}$ in forming gas ambient to ensure good ohmic contacts and passivation at the front and rear device surfaces.

To preserve lifetime all thermal oxidations were performed in very clean conditions, thanks to the use of Dicloroethylene (DCE) in oxidation furnaces. Besides RCA cleaning sequence was used prior to every high-temperature step.

\section{B. Cover Glass Metallization}

We use uncoated cover glass 4" wafers of $400 \mu \mathrm{m}$ thickness (model CMO430 QIOPTIQ ${ }^{\circledR}$ ). The cover glass metallization has been made as follows: a) photoresist deposition at the front side; b) metallization by e-beam evaporation of a stack of Ti/Pd $(30 \mathrm{~nm} / 120 \mathrm{~nm})$; and c) metal patterning using liftoff technique and thermal annealing in inert ambient $\left(\mathrm{N}_{2}\right) \mathrm{T}=105^{\circ} \mathrm{C} t=$ $15 \mathrm{~min}$ to ensure good adherence between metals and cover glass.

\section{Silicon-Cover Glass Bonding}

To bond cover glass with silicon we use the glue bonding technique. To perform the silicon-glass integration the spatial NASA certified transparent non-conductive epoxy EPO-TEK ${ }^{\circledR}$ 302-3M was used. Prior to bonding epoxy was degasified in vacuum (0.02 mbar) to avoid bubbles. The epoxy was deposited carefully in four peripheral points to avoid covering the electrodes (die pads) and windows. The bonding step was performed with a maximum alignment error of $50 \mu \mathrm{m}$. Finally, the epoxy was cured at $\mathrm{T}=65^{\circ} \mathrm{C} t=180 \mathrm{~min}$ in $\mathrm{N}_{2}$ ambient.

\section{Characterization}

In order to be able to assess the optical losses, the light transmittance of cover glass has been measured as it is shown in
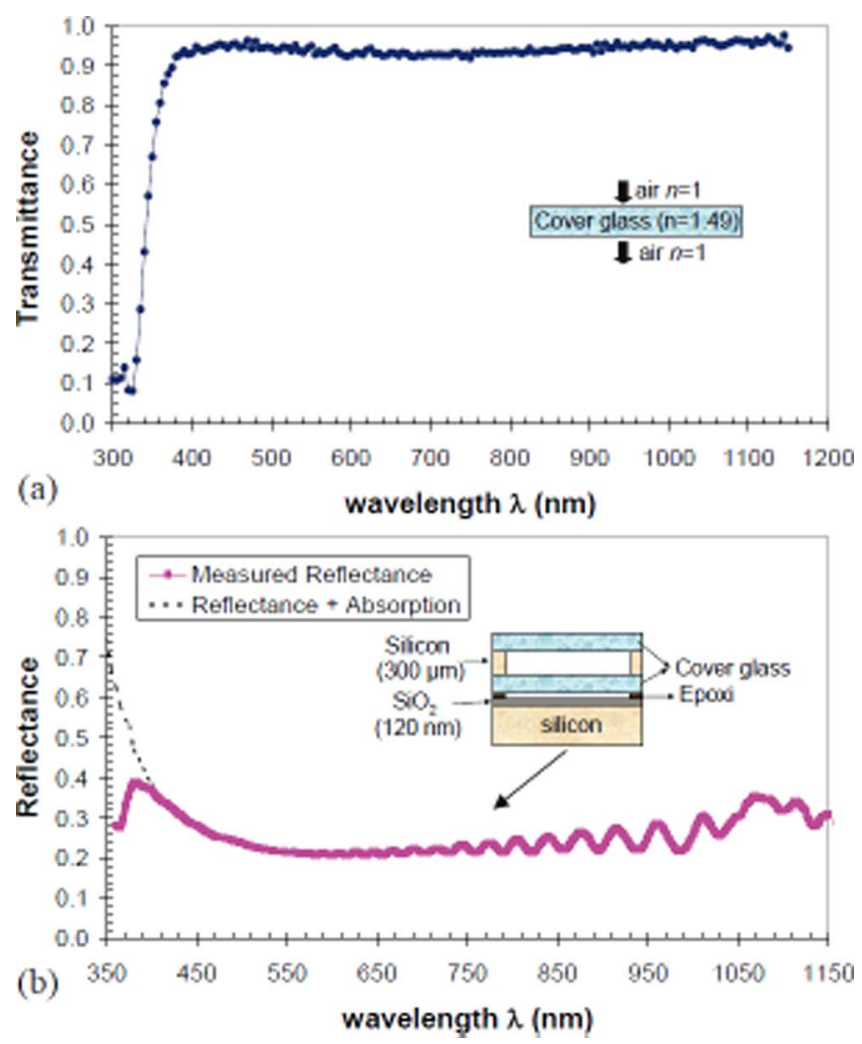

Fig. 5. (a) Light transmittance of a $400 \mu \mathrm{m}$ thick cover glass. (b) Measured reflectance (continuous line) and calculation of the effective reflectance (dashed line) versus wavelength. The reflectance measurement setup is shown in the inset.

Fig. 5(a), yielding transmitance greater than $94 \%$ in the visible and near infrared (wavelengths $\lambda$ 's ranging from 380-1200 nm). However cover glass has a relevant absortance in the ultraviolet part of the spectrum (300-380 nm). Light reflectance at the top surface of our devices was also measured, and an effective reflectance $R_{e f}$ was calculated taking into account the reflectance and cover glass absortance losses as can be seen in Fig. 5(b). To emulate the encapsulated device, as it is explained in Section IV, an extra cover glass is incorporated to the measurements [see measurement setup scheme in the inset of Fig. 5(b)]. Measuring the period of oscillations in the experimental reflectance curve [19], the thickness of epoxy can be estimated, to about $3 \mu \mathrm{m}$.

The electrical behavior of the sensor photodiodes was measured using standard terrestrial test conditions: solar light spectrum AM1.5 $1000 \mathrm{~W} / \mathrm{m}^{2}, \mathrm{~T}=25^{\circ} \mathrm{C}$, and normal incidence. In Fig. 6 the illuminated I-V curves for the two pair of photodiodes are shown. As it can be seen, a very good matching between the curves has been obtained in our devices (differences below $2 \%$ in photocurrents). Main photovoltaic parameters values are included in the graphs (see tables inset in the graphs). It is important to stress here that the parameter $K$ has values ranging from 0.28 to $0.3 \mathrm{~A} / \mathrm{W}$ using spectrum AM1.5 for our devices, confirming that recombination at the front surface and in the bulk is low (see Fig. 2).

\section{ELECTRONICS AND MECHANICAL INTERFACE}

The auxiliary electronics consists basically of four current-voltage converters, one per photodiode as can be seen in 

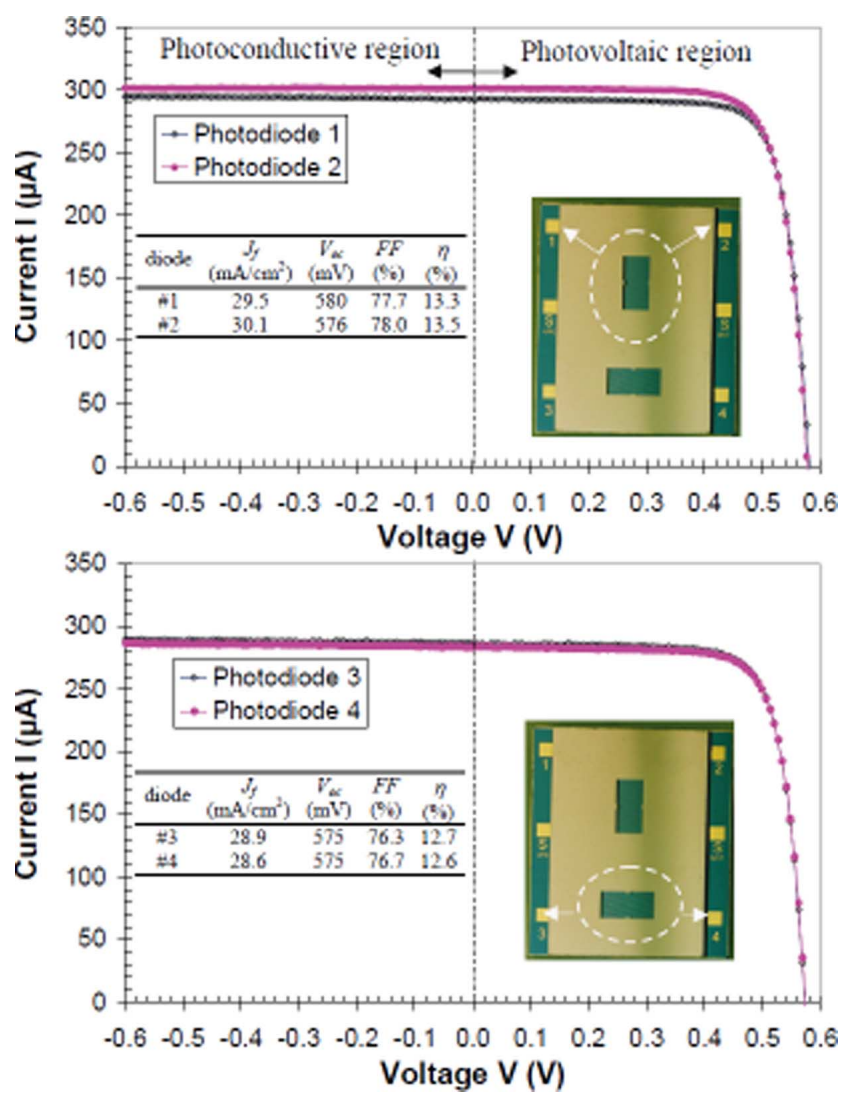

Fig. 6. Illuminated I-V curves for the two photodiodes pairs. Standard AM1.5 $\left(1000 \mathrm{~W} / \mathrm{m}^{2}\right.$ and $\left.\mathrm{T}=25^{\circ} \mathrm{C}\right)$ and perpendicular incidence was used. Tables in the graphs summarize main photovoltaic parameter values, where $J_{f}$ is the photocurrent density (an active area of a $1 \mathrm{~mm}^{2}$ has been considered for every photodiode), $V_{o c}$ is the open circuit voltage, $F F$ the fill factor, and $\eta$ the photovoltaic efficiency.

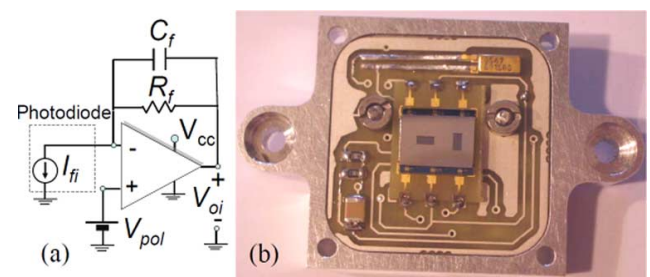

Fig. 7. (a) Simplified current-to-voltage converter circuit for a single photodiode. (b) Image of the integration of the silicon die with the auxiliary electronics. The total size of the printed circuit board PCB with the auxiliary electronics is $2.6 \mathrm{~cm} \times 2.6 \mathrm{~cm}$.

Fig. 7(a). The amplifier gain is adjusted by means of $R_{f}$ resistance taking into account maximum expected current and saturation output voltage level of amplifiers. A voltage offset $V_{p o l}$ is established in the non-inverting input of amplifier to polarize photodiodes in the photoconductive region, where current is almost constant an equal to $I_{f}$. The capacitor $C_{f}$ is used to reduce high frequency noise. Discrete components and the amplifier used (LM124AWGRL-QMLV) fulfil the space standards MIL-PRF-38535 QML Level V 5962-99054. Fig. 7(b) shows the implementation of the printed circuit board containing the solar sensor and its auxiliary electronics. Electronics and silicon sensor are packaged in an alodined $3 \mathrm{~mm}$ thickness aluminum box to attenuate the influence of the outer-space radiation effect.

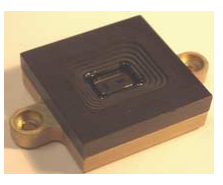

Fig. 8. Sensor implementation. Total volume is $3.0 \mathrm{~cm}$ (width) $\times 3.0 \mathrm{~cm}$ (length) $\times 1.2 \mathrm{~cm}$ (height).

Assuming an ideal behavior of the current-voltage stages and the same feedback $R_{f}$ resistance in the four amplifiers, we can obtain $R_{\theta}$, for instance, combining the output voltages of a photodiode pair as follows:

$$
R_{\theta}=\frac{V_{o 1}-V_{o 2}}{V_{o 1}+V_{o 2}-2 V_{p o l}}=\frac{I_{f 1}-I_{f 2}}{I_{f 1}+I_{f 2}} .
$$

These amplified voltages are the inputs to the Attitude and Orbit Control Subsystem (AOCS) of the satellite platform, which carries out the digital processing of the signals providing the incidence angles of solar radiation.

The electronics assembly has been done considering the special requirements demanded by space applications. One of these requirements [15] determines that golden terminals, where welding processes involve Sn, shall not be used. For this reason, the solar sensor has been wire-bonded to an intermediate board $(1.3 \mathrm{~cm} \times 1.05 \mathrm{~cm})$ with golden terminals using $25 \mu \mathrm{m}$ gold wires to facilitate this process, using double wire to have a redundant electrical connection in each pad. Its pads in the periphery have been de-golden by mechanical polishing following recommendations given in [15]. This intermediate board has been glued and welded on top of a double-sided printed circuit board PCB $(2.6 \mathrm{~cm} \times 2.6 \mathrm{~cm})$ with pretinned wires. The layout of the electronic components has been determined according to its functionality and maximizing their protection against high energy particle radiation. The silicon sensor is placed in the center of the board where a window in the aluminum package is located. The sensor is protected with an additional external cover-glass placed on the package. The rest of the other components are located near the edges of the back side of the board, as far from the input window as possible. In Fig. 8, the sun sensor is shown once auxiliary electronics and silicon die are enclosed in the aluminum box. The steps in the input window of the aluminum structure are designed to avoid light reflections inside the active area of the sensor.

It should be stressed that all previous manufacturing steps above described have tolerances, and a final misalignment is expected respect to the sensor positional reference. For this reason, a ground calibration of the sensor, as it will be explained in the next section, is necessary to compensate these deviation effects.

\section{RESULTS}

\section{A. Angle Measurements}

Sensor calibration has been performed at the Laboratory of Certification of photovoltaic solar cells for space use, SPASOLAB, located in the Instituto Nacional de Técnica Aeroespacial (INTA). This calibration procedure consists in the use of a high-accurate Angular Positioning System (APS), see Fig. 9, specifically developed by the USE group for this purpose. This 


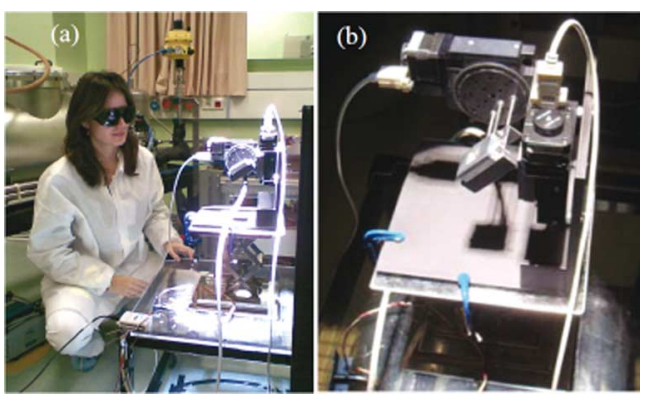

Fig. 9. (a) Calibration of a sun sensor in the INTA Spasolab laboratory using a solar simulator (AM0 spectrum $1366 \mathrm{~W} / \mathrm{m}^{2}$ of irradiance). (b) A detail of the two-axis rotary motorized table instrument.

instrument is necessary to achieve high precision calibration curves, and to compensate any misalignment suffered by the sensor in their manufacturing steps. The APS consists in two rotary stepper motors, whose axes cut in the centre of the sensor to be calibrated. In this way, the displacement of the sensor below the radiation field is limited and the negative effect of the nonuniformity of the radiation is reduced. STANDA 8MR191 Stepper motors have been used for this table because their precision of $0.01^{\circ}$ is more than one order of magnitude better that the desired target precision. Before calibrating a sensor, the table is placed below a solar simulator and a reference solar cell is used to adjust the standard AM0 irradiance $\left(1366 \mathrm{~W} / \mathrm{m}^{2}\right)$. The positioning table is controlled using specific programs implemented with Labview v8.0. This software generates a sun sensor calibration pattern that forms a $5^{\circ} /$ step matrix when moving both incident angles from $-60^{\circ}$ to $60^{\circ}$. Proprietary software characterizes and postprocesses the response of the unit and generates the corresponding calibration tables.

An example of the voltage measured in a particular photodiode as a function of the light incident angles $\theta_{i}$ and $\phi_{i}$ can be seen in Fig. 10(a). In this example, the existence of a roughness in the surface can be appreciated. Unfortunately, this effect was produced for the bubbles that appeared in the epoxy resin during the wire bonding stage (see photograph in Fig. 10(a)). This is the reason why it was decided to place resin out of the sensing areas of the photodiodes. As result, smooth surfaces, like the one depicted in Fig. 10(b), were obtained.

The next step in the calibration procedure is the calculation of $R_{\theta}$ and $R_{\phi}$ as functions of incident angles $\theta_{i}$ and $\phi_{i}$. As an example, an experimental function $R_{\theta}\left(\theta_{i}, \phi_{i}\right)$ is shown in Fig. 11. This experimental surface is quite close to the theoretical one presented in Fig. 3.

Once the calibration curve is obtained it is necessary a verification process, in which the sensor is tested in the two-axis rotary motorized table. Fig. 12 shows the errors between the predicted angles using the calibration curve and the actual ones for a particular sun sensor. The sensor response for 450 random positions are captured and compared with the real angles provided by steppers. We can see that errors in the $99.5 \%$ of angle positions are smaller than $0.1^{\circ}$, and errors smaller than $0.05^{\circ}$ are in the $87.3 \%$ of measurements. Thus, the mission requirements as a FOV of $\pm 60^{\circ}$ and angle resolution better than $0.5^{\circ}$, about $0.15^{\circ}$ in our sun sensors, have been successfully achieved.

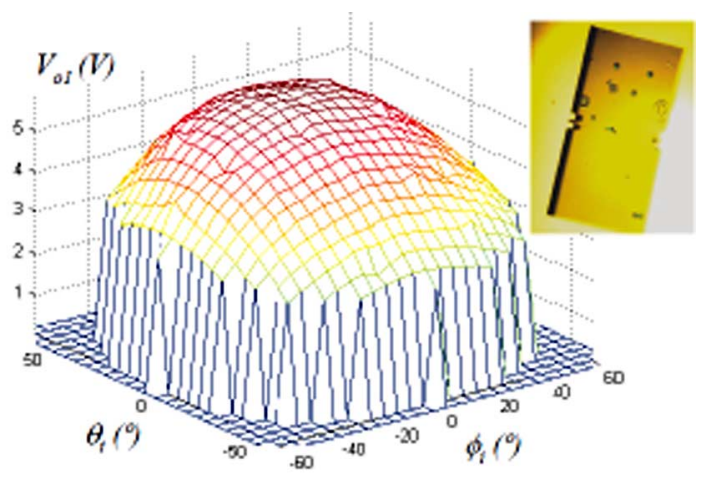

(a)

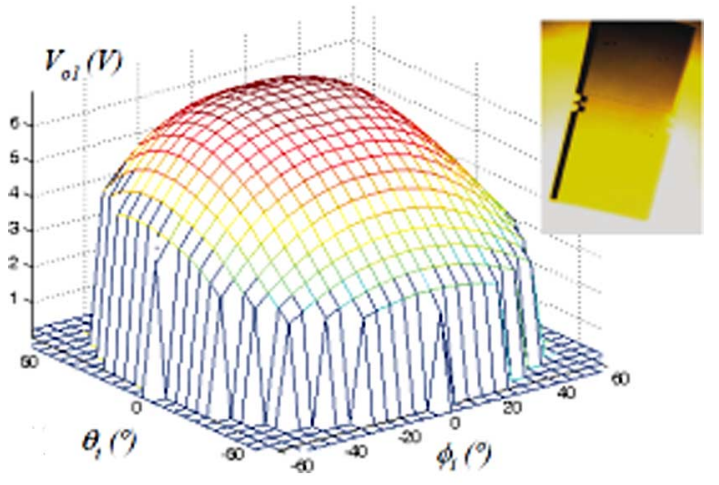

(b)

Fig. 10. (a) Surface representing the voltage measured in a cell (photodiode 1) versus the incident angles of the light. (b) A view through one of the windows in the cover-glass where it can be appreciated some bubbles that were formed during the wire bonding stage that caused the nonuniformities in the calibration surface.

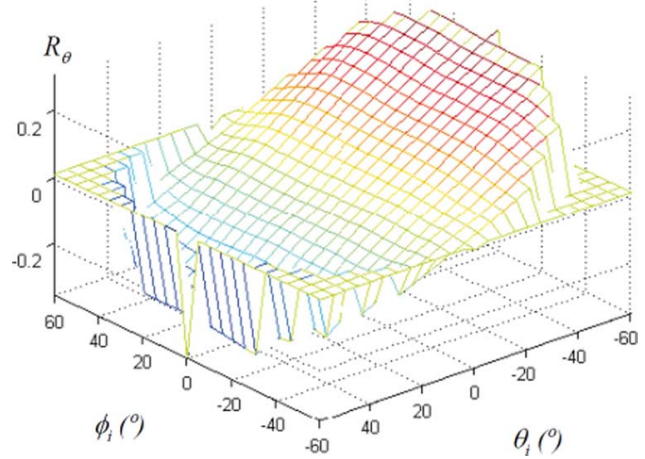

Fig. 11. Representation of the experimental calibration surface $R_{\theta}$ as a function of the incident angles $\theta_{i}$ and $\phi_{i}$. A FOV of $\pm 60^{\circ}$ has been considered $\left(\varphi_{i} \leq 60^{\circ}\right)$.

\section{B. Flight Qualification Tests}

Sensors have been subjected to a set of qualification tests in order to guarantee the necessary life-cycle of NANOSAT-1B mission (see [20] for details). Outgassing, thermal, vibration, EMI and impact tests of sun sensors have been carried out using INTA facilities. A qualification model (QM) prototype has been tested with vibrations of $10 \mathrm{~g}$ at frequencies up to $1 \mathrm{KHz}$ and shocks, with accelerations range 50 to $30000 \mathrm{~g}$ from 1.0 to $0.12 \mathrm{~s}$, respectively. Destructive effects cannot be observed by means of the sounds produced in hazard 


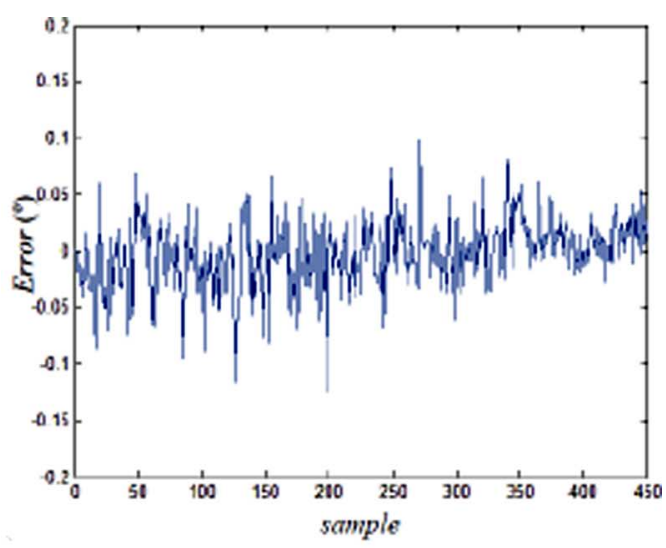

(a)

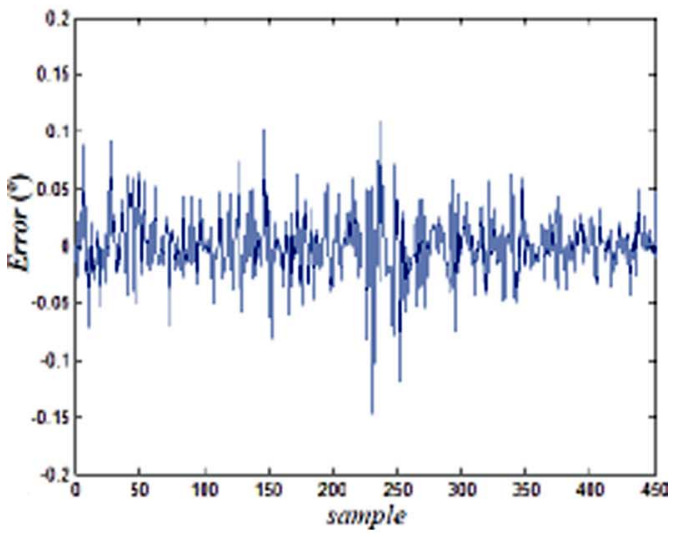

(b)

Fig. 12. Errors between actual and measured (a) $\theta_{i}$ and (b) $\phi_{i}$ angles, for 450 random positions in the whole FOV.
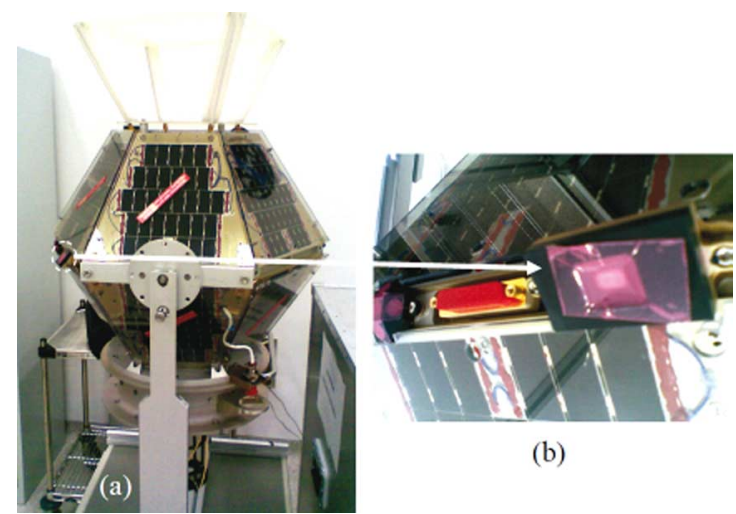

(b)

Fig. 13. (a) Flight Model (FM model) of the NANOSAT-1B satellite. (b) A detail of one of the tree sensors once integrated in the platform.

shocks throughout vibration test. Thermal test involves a sharp warming. Sun sensors have been exposed to 50 thermal cycles of $120^{\circ} \mathrm{C}$ amplitude temperature increasing at $10^{\circ} / \mathrm{min}$ rates. It has not been observed changes in colors or permanent deformations that might indicate degradation in the selected component. Some Sun sensors were irradiated in a Tandem Van de Graaff particle accelerator, being exposed to orbit radiation conditions of the NANOSAT-1B mission, without observing any degradation in the sensor structure and functionality.
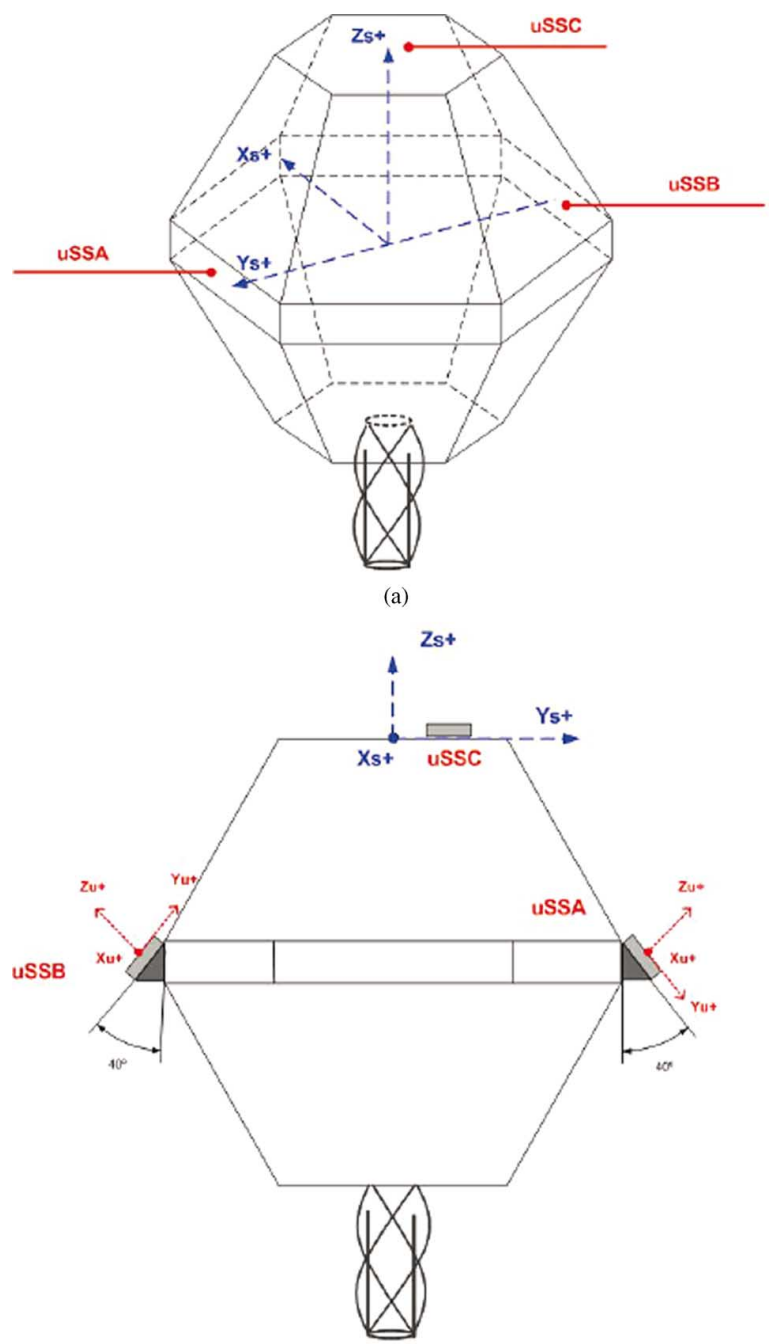

(b)

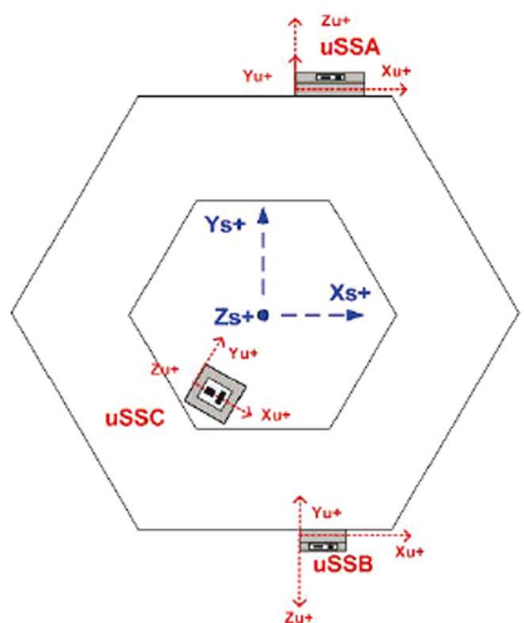

(c)

Fig. 14. NANOSAT-1B satellite body with the location of the three sun sensors (uSSA, uSSB and uSSC): (a) 3D view, (b) side view, and (c) top view.

\section{Satellite Integration}

The sun sensors are part of the Complementary Scientific Payload of Spanish nano-satellite NANOSAT-1B (see Fig. 13), manufactured by the INTA, at launched in July 2009. They provide support to the AOCS determining the two-axis incidence 
angle of a solar radiation, in order to achieve the on-orbit attitude satellite control.

Three sun sensors (uSSA, uSSB, and uSSC) have been installed in the satellite as it is depicted in Fig. 14, where $\left(\mathrm{X}_{u}, \mathrm{Y}_{u}, \mathrm{Z}_{u}\right)$ are the coordinate systems respect to each sensor, while $\left(\mathrm{X}_{s}, \mathrm{Y}_{s}, \mathrm{Z}_{s}\right)$ is the inertial reference system respect to satellite body

The orientation of the three sensors has been calculated to guarantee that at least one sensor receives sun light during its normal operation when $Z_{s}$ is opposite to nadir pointing. Two $40^{\circ}$ wedges have been accommodated in uSSA and uSSB bases to prevent sensors from albedo effects. Transformations of coordinates from sensors to the satellite reference system are given by

$$
\begin{aligned}
& \left(\begin{array}{l}
X_{s} \\
Y_{s} \\
Z_{s}
\end{array}\right)=\left(\begin{array}{ccc}
1 & 0 & 0 \\
0 & \cos 50^{\circ} & \sin 50^{\circ} \\
0 & -\sin 50^{\circ} & \cos 50^{\circ}
\end{array}\right)\left(\begin{array}{l}
X_{u}^{A} \\
Y_{u}^{A} \\
Z_{u}^{A}
\end{array}\right) \\
& \left(\begin{array}{c}
X_{s} \\
Y_{s} \\
Z_{s}
\end{array}\right)=\left(\begin{array}{ccc}
1 & 0 & 0 \\
0 & \cos 50^{\circ} & -\sin 50^{\circ} \\
0 & \sin 50^{\circ} & \cos 50^{\circ}
\end{array}\right)\left(\begin{array}{l}
X_{u}^{B} \\
Y_{u}^{B} \\
Z_{u}^{B}
\end{array}\right) \\
& \left(\begin{array}{c}
X_{s} \\
Y_{s} \\
Z_{s}
\end{array}\right)=\left(\begin{array}{ccc}
\cos 30^{\circ} & \sin 30^{\circ} & 0 \\
-\sin 30^{\circ} & \cos 30^{\circ} & 0 \\
0 & 0 & 1
\end{array}\right)\left(\begin{array}{l}
X_{u}^{C} \\
Y_{u}^{C} \\
Z_{u}^{C}
\end{array}\right) .
\end{aligned}
$$

Nowadays, sun sensors data and telemetries are being received on ground station successfully. The analysis of the information received suggests that flight sun sensors fulfill the resolution, accuracy and functional targets of the mission.

\section{CONCLUSION}

This paper shows the design, fabrication, characterization, and satellite integration of a miniaturized two-axis sun sensor for satellite attitude control. This device uses monolithically integrated four silicon photodiodes, including a transparent cover glass on the same silicon die to act as shield to prevent space radiation damage. Device fabrication combines microelectronics technology with a high efficiency solar cell fabrication process, allowing a small size $(3 \mathrm{~cm} \times 3 \mathrm{~cm})$ and low weight $(24 \mathrm{gr})$ device. Our sun sensors fulfill mission requirements yielding a sun FOV upper $\pm 60^{\circ}$, and angle accuracy better than $0.15^{\circ}$. Once the qualification prototype pass the vibration, thermal and radiation tests, three flight devices have been mounted in the Spanish nano-satellite NANOSAT-1B that has been successfully launched in July of 2009.

\section{ACKNOWLEDGMENT}

The authors wish to thank to the Centro Nacional de Aceleradores (CNA), Seville, for the radiation tests, as well as the technical staff of the Clean Room Laboratory, UPC, for their technical assistance to fabricate the silicon dice. They would like to express special thanks to the INTA for using their facilities to test and qualifying our devices as well as the INTA team for their reliable good work in the integration of our sun sensors in the NANOSAT-1B satellite.

\section{REFERENCES}

[1] X. S. Ge and L. Q. Chen, "Attitude control of a rigid spacecraft with two momentum whell actuators using genetic algorithm," Acta Astronautica, vol. 55, pp. 3-8, 2004.

[2] M. Wood, W. Chen, and D. Fertin, "Model predictive control of low earth orbiting spacecraft with magneto-torquers," in Proc. IEEE Int. Conf. Control Applications, Munich, Germany, Oct. 2006, pp. 2908-2913.

[3] Y. Zhang, Y. Postrekhin, K. B. Ma, and W. K. chu, "Reaction whell with HTS bearings for mini-satellite attitude control," Supercond. Sci. Technol., vol. 15, pp. 823-825, 2002.

[4] D. Tong, "Spacecraft momentum dumping using gravity gradient," $J$. Spacecraft and Rockets, vol. 35, pp. 714-717, 1998.

[5] Y. W. Jan and J. C. Chiou, "Attitude control system for ROCSAT-3 microsatellite: A conceptual design," Acta Astronautica, vol. 56, pp. 439-452, 2005.

[6] A. W. van Herwaarden, "Low-cost satellite attitude control sensors based on integrated infrared detector arrays," IEEE Trans. Instrum. Meas., vol. 50, no. 6, pp. 1524-1529, Dec. 2001.

[7] M. M. Birnbaum, "Spacecraft attitude control using star field trackers," Acta Astronautica, vol. 39, pp. 763-773, 1996.

[8] Y. K. Chang, M. Y. Yun, and B. H. Lee, "A new modelling and validation of two-axis miniature fine sun sensor," Sens. Actuators A: Phys., vol. 134 , pp. $357-365,2007$

[9] J. H. Hales and M. Pedersen, "Two-axis MOEMS sun sensor for pico satellites," in Proc. 16th Аnnu. AIAA/USU Conf. Small Satellites, Logan, UT, Aug. 2002, pp. 1-12.

[10] T. Böhnke, M. Edoff, and L. Stenmark, "Development of a MOEMS sun sensor for space applications," Sens. Actuators A: Phys., vol. 130-131, pp. 28-36, 2006.

[11] J. M. Quero, C. Aracil, L. G. Franquelo, J. Ricart, P. R. Ortega, M. Dominguez, L. Castañer, and R. Osuna, "Tracking control system using an incident radiation angle microsensor," IEEE Trans. Ind. Electron., vol. 54, no. 2, pp. 1207-1215, Apr. 2007.

[12] Hamamatsu Photonics Data Manual, s6560 Product, [Online]. Available: http://www.hamamatsu.com

[13] C. C. Liebe, "Solar compass chip," IEEE Sensors J., vol. 4, no. 6, pp. 779-786, Dec. 2004.

[14] "Data for selection of space materials and processes of space product assurance," European Cooperation for Space Standardization, ECSS-70-71A, 2004

[15] "The manual soldering of high-reliability electrical connections," European Cooperation for Space Standardization, ECSS-Q-70-08A, 1999.

[16] J. Rusell, G. Jones, and J. Hall, "A new UVR/IRR coverglass for triple junction cells," in Proc. 4th IEEE World Conf. Photovoltaic Energy Conversion, Waikoloa, HI, May 2006, pp. 1911-1914.

[17] D. Clugston and P. Basore, "PC-1D version 5: 32 bit solar cell modelling on personal Computers," in Proc. 26th IEEE Photovoltaic Specialist Conf., Anaheim, CA, Sep.-Oct. 1997, pp. 207-210.

[18] P. Ortega, S. Bermejo, and L. Castañer, "High voltage photovoltaic mini-modules," Progress in Photovoltaics: Res., vol. 16, pp. 369-377, 2008.

[19] R. Swanepoel, "Determination of the thickness and optical constants of amorphous Silicon," J. Phys. E: Scientific Instruments, vol. 16, pp. 1214-1222, 1983.

[20] J. G. Ortega, C. L. Tarrida, J. M. Quero, F. J. Delgado, P. Ortega, L. Castañer, M. Reina, M. Angulo, Y. Morilla, and J. G. López, "Mems solar sensor testing for satellite applications," in Proc. IEEE Spanish Conf. Electron Devices, Santiago de Compostela, Spain, Feb. 2009, pp. 345-348.

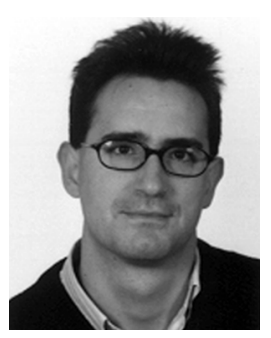

Pablo Ortega received the M.Sc. degree and the $\mathrm{Ph} . \mathrm{D}$. degree in ingeniería de telecomunicación from the Universidad Politécnica de Cataluña (UPC), Barcelona, Spain, in 1991 and 2000, respectively. His $\mathrm{Ph} . \mathrm{D}$. dissertation research work focused on the development of new packaging strategies for photovoltaic mini-modules.

Currently, he is an Associate Professor at the UPC, Micro \& Nano Technologies Group (MNT). His research interest involves design, fabrication, characterization and simulation of solar cells and photovoltaic devices based on crystalline silicon, as well as the study of new applications based on photovoltaic energy. 


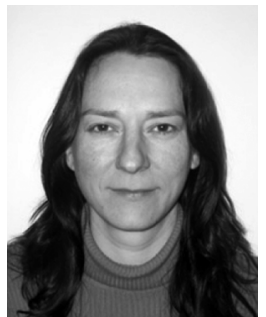

Gema López-Rodríguez received the M.Sc. degree in electronic engineering from the Universidad Politécnica de Cataluña (UPC), Barcelona, Spain, in 2008.

She joined the Micro and Nanotechnologies Group, Electronic Engineering Department, UPC, in 2006, as a Research Assistant. Since 2006, she is working in solar cells research, silicon micromachining devices, and sensor technology applied to space applications.

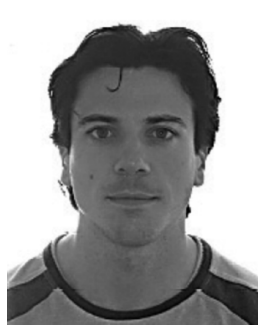

Jordi Ricart received the M.Sc. degree in ingeniería electrónica from the Universidad Politécnica de Cataluña (UPC), Barcelona, Spain, in 2005, where he is currently working towards the Ph.D. degree.

$\mathrm{He}$ joined the Micro and Nano Technologies Group, Electronic Engineering Department, UPC, as both a Research Assistant and a Ph.D. student. He has worked in sensors for photovoltaic processes, thermal flow meter fabrication for martian atmosphere. His research interests also include the design of pulsed oscillators for MEMS devices.

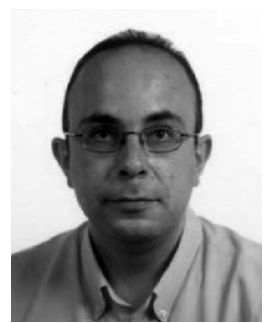

Manuel Dominguez received the M.Sc. and Ph.D. degrees in electronic engineering both from the Universidad Politécnica de Cataluña (UPC), Barcelona, Spain, in 1994 and 1997, respectively, and the Degree in mathematics (Hon) from the Universidad Nacional de Educación a Distancia (UNED), Madrid, Spain, in 2005.

$\mathrm{He}$ has been with the Department of Electronic Engineering, UPC, since 1994, where he is now an Associate Professor. He was a Visiting Scholar at the Courant Institute of Mathematical Sciences from September 2006 to August 2007. He has participated in the design of a 3-D anemometer for Mars atmosphere within the REMS project of the Mars Science Laboratory, NASA. His research areas include the design and development of MEMS sensors and actuators, sensors for space applications, sigma-delta modulation applied to MEMS, oscillators, and nonlinear circuits in general.

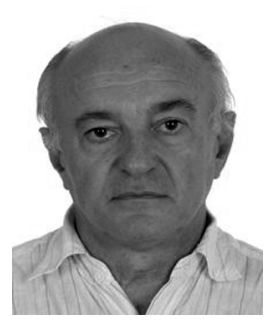

Luis M. Castañer (M'77-SM'92) received the Doctor Ingeniero de Telecomunicación from the Departamento de Ingeniería Electrónica de la Escuela Técnica Superior de Ingenieros de Telecomunicación, Universidad Politécnica de Cataluña (UPC), Barcelona, Spain, following his undergraduate studies, Ingeniero Superior de Telecomunicación from the Universidad Politécnica de Madrid, Madrid, Spain, in 1971, and the Diplôme d'Etudes Approfondies (D.E.A.) en Physique Spatiale and Docteur-Ingenieur from the Université Paul Sabatier,

Toulouse, France, 1973.

He is a Professor with the Departamento de Ingeniería Electrónica de la Escuela Técnica Superior de Ingenieros de Telecomunicación, UPC. He has also been Dean of E.T.S.I. Telecomunicación and Head of the Departamento de Ingeniería Electrónica, and has held several positions in Research Agencies and Committees: representative in the Comite de Gestion y Coordinacion on non-nuclear energies, DGXII Comission of the EU, Head of the Programa Nacional de Tecnologías de la Información y Comunicaciones of the CICYT (1992-1994), and Coordinator of the Technology Foresight at the Agencia Nacional de Evaluación y Prospectiva. He has contributed to semiconductor device research, covering solar cells in various aspects: technology of CIS, space degradation of Silicon and GaAs devices, and has contributed to the theory and technology of advanced bipolar transistors with polysilicon emitters and its application to high efficiency silicon solar cells, in particular, to the emitter resistance of these devices. He has also contributed to the design, simulation and monitoring of photovoltaic power plants and systems, and is active in the microsystems technology area, working on flow sensors, dielectric degradation of MEMS, electrostatic actuators, and wind sensors for Mars.

Prof. Castañer is a Member of the Association and Charter of Telecommunication Engineers in Spain, and a Member of the Royal Spanish Engineering Academy.

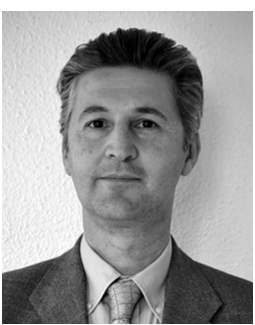

José M. Quero (M'97-SM’06) was born in Seville, Spain, in 1963. He received the M.Sc. and Ph.D. degrees in electrical engineering from the University of Seville, Seville, Spain, in 1988 and 1991, respectively.

In 1988, he joined the Department of Electronic Engineering, University of Seville, as an Assistant Professor. He became an Associate Professor in 1992 and, since 2000, he has been a Full Professor. He teaches analog and digital microelectronics and $\mathrm{mi}-$ crosystems in the Engineering School, University of Seville. He is also Senior Researcher within AICIA, a non-profit research organization. He is evaluator and reviewer for the European Commission in the Information Society Technology (IST) programme since 2002. Currently, he is CEO of the spin-off company Solar MEMS Technologies. His research interests include MEMS sensors and actuators and their application in medicine, microfluidics and space.

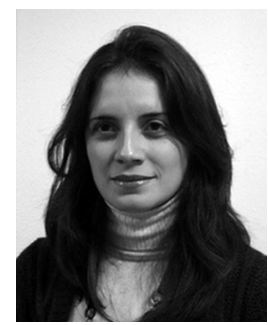

Cristina L. Tarrida was born in Seville, Spain, in 1979. She received the B.S. degree in industrial engineering from the University of Seville, Seville, Spain, in 2007.

From 2004 to 2009, she has been a Technical Specialist in the Department of Electronic Engineering, University of Seville. Currently, she is Engineering Manager of the spin-off company Solar MEMS Technologies S.L. Her research interests include MEMS applied to renewable energies, energy efficiency, and space areas.

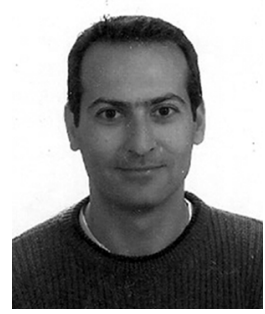

Juan García was born in Seville, Spain, in $1965 . \mathrm{He}$ received the $\mathrm{Ph} . \mathrm{D}$. degree in electrical engineering from the University of Seville, Seville, Spain, in 1995.

In 1993, he was Researcher in the Department of Electronic Engineering, University of Seville, and in 2001, he became an Assistant Professor. He is currently teaching several courses on semiconductors and MEMS technology in telecommunication and aeronautic engineering. He has participated in many research projects and collaborations with local and regional industry. His research areas include laser micromachining and MEMS sensors in aerospace applications.

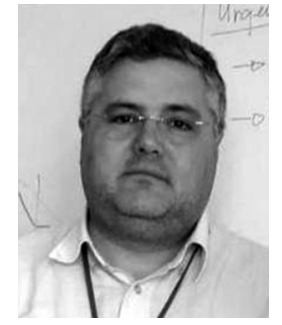

Manuel Reina received the M.S degree in aeronautic engineering from the Universidad Politécnica de Madrid (UPM), Madrid, Spain, in 1989.

Since 1989, he has been with INTA as a Thermal Engineer of Space Equipment and Test Engineer. Since 2000, he has been the Head of the Engineer Group within the Payloads and Space Instruments Area. He has been involved with Space Instruments such as FWM OSIRIS (a mechanism for ROSETTA spacecraft), IMaX a Solar magnetospectometer, and INTA NANOSAT satellites. 


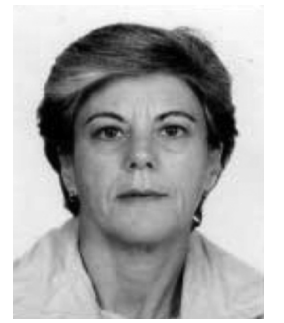

Ana Gras received the M.Sc. degree in applied physics from The John Hopkins University, Baltimore, MD, in 1997 and the M.Sc. degree in astrophysics from the Universidad Complutense, Madrid, Spain, in 1981.

She has worked at the Instituto de Energía Solar (IES), Madrid, and at the European Space Technology Centre (ESTEC) in the European Space Agency (ESA). In 1986, she joined the Instituto Nacional de Técnica Aeroespacial (INTA) as Technical responsible for setting up SPASOLAB, a laboratory for qualification and evaluation of solar cells for space applications. Currently, her activity at SPASOLAB involves research and development of new test methods for characterization of emergent photovoltaic devices for space applications.

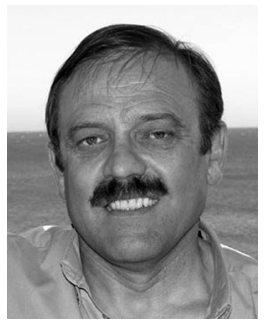

Manuel Angulo received the Aeronautical Technical Engineering degree from the Universidad Politécnica de Madrid, Madrid, Spain, in 1972, and the M.S. degree in physics from the Universidad Nacional de Educación (UNED, Madrid, in 1987.

Currently, he is responsible for the nano and microsatellites programs at the Instituto Nacional de Técnica Aeroespacial (INTA). In 1976, he joined the Institute and has been engaged in many space activities starting with the development of atmospheric sounding rockets (INTA-100) for eight years, and stratospheric balloon scientific payloads crossing the Mediterranean basin from Italy to Spain. Later on in 1985, he joined the Avionics Department and worked in the development of several space electronic units for ESA missions and other European satellite programs. During the period 1988-1991 and phases 0 , A \& B, he was Project Manager (PM) of the Spanish satellite Minisat-01, later on launched by a Pegasus above the Canary Islands in 1997. From 1992 to 2000, he participated in several feasibility studies for ESA and other Spanish space programs. In 2001, he was appointed PM of the Nanosat-1 satellite (19 $\mathrm{Kg}$ ), finally launched by an Ariane-5 in December 2004 from Kourou, French Guiana. During the last six years, he also put in orbit the follow-on mission Nanosat-1B $(24 \mathrm{Kg})$, launched by a Dnepr form Baikonur, Kazakhstan. Both missions are performing store and forward communications in the UHF band, together with several scientific experiments in the field of nanotechnology, microtechnolgy, and MEMS. He is currently working as a PM on a new mission called INTAmSat-1 with $110 \mathrm{Kg}$, that will be the first Earth R\&D observation satellite fully developed in Spain. 